\title{
Deixar a pátria livre ou morrer pelo Haiti: sobre a global governance no caso da MINUSTAH ${ }^{1}$
}

\section{Leave a Free Country or Die for Haiti: On Global Governance in the Case of MINUSTAH}

\author{
Carlos Enrique Ruiz Ferreira ${ }^{2}$
}

Universidade Estadual da Paraíba (Brasil)

Daniel Afonso da Silva ${ }^{3}$

Ceri-Sciences Po (Francia)

Marília Cordeiro Serra ${ }^{4}$

Universidade Estadual da Paraíba (Brasil)

Recibido: 18-06-16

Aprobado: 03-02-17

\section{Resumo}

Deixar a pátria livre ou morrer pelo Haiti aborda a global governance a partir do caso da MINUSTAH - Mission des Nations Unis pour la stabilization

\footnotetext{
${ }^{1}$ Este artigo contou com o apoio e financiamento da Coordenação de Aperfeiçoamento de Pessoal em Nível Superior (CAPES) e do Projeto "A Política Externa Brasileira no Conselho de Segurança da ONU no Pós Guerra Fria", aprovado no Edital CNPq/UNIVERSAL/14/2013 do Ministério de Ciência e Tecnologia da República Federativa do Brasil. Os autores agradecem ao Professor Luis Ochoa Bilbao, da Benemérita Universidad Autónoma de Puebla, pelo incentivo, pela confiança e pela consideração e aos alunos do Bacharelado e do Mestrado em Relações Internacionais da Universidade Estadual da Paraíba, turmas 2013 e 2014, que viram nascer as primeiras impressões deste artigo.

2 (carloserf@uol.com.br) Doutor em Ciências Políticas pela Universidade de São Paulo e professor de Relações Internacionais da Universidade Estadual da Paraíba. Autor de Professor Oliveiros S. Ferreira: Brasil, Teoria Política e Relações Internacionais em sua obra (São Paulo: Edusp, 2015) e, em parceria com Daniel Afonso da Silva, Celso Luiz Nunes Amorim: entre virtudes e vocações (Campina Grande: Eduepb, 2017).

3 (daniel.afonso66@hotmail.com) Doutor em História Social pela Universidade de São Paulo, pósdoutor em Relações Internacionais pelo Centre de Recherches Internationales da Sciences Po de Paris e pesquisador do Núcleo de Pesquisas em Relações Internacionais da Universidade de São Paulo. Autor de Était de lieux du malaise brésilien (In: Jaffrelot, Christophe. (Org.). Les BRICS : chronique d'une mort annoncée, mais sans cesse reportée. 1ed.Paris: Les dossier du CeriSciences Po, 2015), Después de los ojos azules: Lula da Silva, le moment brésilien y la imaginación nacional brasileña (In. : Ochoa, Luis; Rodrígez, Myrna; Pneau, Marisa. Los BRICS y el discurso del nacionalismo en el siglo XXI. Washington: Westphalia Press, 2015) e, em parceria com Carlos Enrique Ruiz Ferreira, Celso Luiz Nunes Amorim: entre virtudes e vocações (Campina Grande: Eduepb, 2017).

${ }^{4}$ (marilia.serra@hotmail.com) Mestre em Relações Internacionais pela Universidade Estadual da Paraíba (Brasil) e professora de Relações Internacionais do Universidade Estácio de Sá em Pernambuco.
} 
en Haiti. Analisa, essencialmente, os relatórios do secretário geral das Nações Unidas produzidos entre 2004 e 2014. Reconstitui o processo de desestabilização política e social que conduziu à necessidade dessa missão no Haiti. Leva em consideração a sobreposição de crises locais, regionais e mundiais nesses dez anos e considera a manutenção como genuíno esforço de global governance.

Palavras-chave: Haiti, MINUSTAH, Global Governance.

\begin{abstract}
Leave a free country or die for Haiti address the global governance from the case of MINUSTAH - Mission des Nations Unis pour la stabilization en Haiti. Analyzes essentially the UN Secretary General's reports produced between 2004 and 2014. Reconstitutes the process of political and social unrest that led to the need of the mission in Haiti. It takes into account the overlap of local, regional and global crises in these ten years and considers the maintenance effort as genuine global governance.
\end{abstract}

Key-words: Haiti, MINUSTAH, Global Governance.

Our Organization, as an organization, was built for a different era. Kofi Annan ${ }^{5}$

Das diversas peacekeeping operations em curso $^{6}$, a MINUSTAH Mission des Nations Unis pour la stabilization en Haiti representa uma das mais conhecidas e discutidas por ser das mais notáveis, longevas e complexas.

\footnotetext{
${ }^{5}$ A/59/2005 - In Larger Freedom: Towards Security, Development and Human Rigths for All Report of the Secretary-General. United Nations, 2 de março de 2005. Parágrafo 154.

${ }^{6}$ MINURSO - The United Nations Mission for the Referendum in Western Sahara, de 29 de abril de 1991. MINUSCA - Multidimensional United Nations peacekeeping operation for Central African Republic, de 10 de abril de 2014. MINUSMA - The United Nations Multidimensional Integrated Stabilization Mission in Mali, de 25 de abril de 2013. MINUSTAH - The United Nations Stabilization Mission in Haiti was established on 1 June 2004. MONUSCO - United Nations Organization Mission in Democratic Republic of the Congo, de 1 de julho de 2010. UNAMID - The African Union/UN Hybrid operation in Darfur de 31 de julho de 2007. UNAMA - The United Nations Assistance Mission in Afghanistan, de 2002. UNDOF - The United Nations Disengagement Observer Force, de 31 de maio de 1974. UNFICYP - United Nations Peacekeeping Force in Cyprus, de 1964. UNIFIL - United Nations Interim Force in Lebanon, de 1978. UNISFA - United Nations Interim Security Force for Abyei, de 27 de junho de 2011. UNMIK - United Nations Interim Administration Mission in Kosov, de 10 de junho de 1999. UNMIL - United Nations Mission in Liberia, 19 de setembro de 2003.UNMISS - United Nations Mission in the Republic of South Sudan, de 8 de julho de 2011. UNMOGIP - United Nations Military Observer Group in India and Pakistan, 24 de janeiro de 1949. UNOCI - United Nations Operation in Côte d'Ivoire, 4 de abril de 2004. UNTSO - United Nations Truce Supervision Organization, Helping to bring stability in the Middle East, maio de 1948.
} 
Recomendada pelo secretário geral das Nações Unidas em 16 de abril de $2004^{7}$ e instaurada sob os auspícios da resolução $1542(2004)^{8}$ saída da reunião do Conselho de Segurança de 30 de abril do mesmo ano, a operação ultrapassa seu primeiro decênio envolvendo mais de meia centena de países e várias dezenas de milhares de recrutas civis e militares de todo o mundo o que faz dela um dos mais formidáveis exemplos de prática de global governance em tempos contemporâneos (Cunliffe 2009 : 323-336).

Desde 2004, o Haiti e o mundo contemplaram diversos momentos de crise e de ordenamentos de superação de crises. Os fantasmas da war on terror do presidente Bush e a brutalidade dos impactos da crise financeira de 2007-2009 começaram a corroer princípios, sentimentos e fundamentos de dignidade e solidariedade por toda parte (Fontana I Làzaro 2011 : 931-963; Ferguson 2013; Mearsheimer 2014; Fukuyama 2014; Badie 2014 ; Dejammet 2015). A fratura social e a insuficiência institucional viraram a marca de emerging, rising e major powers em todos os continentes (Fontana I Làzaro 2013; da Silva 2015; da Silva 2014). Gaza, Iraque, Síria, Ucrânia, Líbia, Sahel, crise econômica, epidemia de Ebola, imigração, terrorismo, Estado Islâmico, ascensão de partidos de extrema direita, preocupações climáticas, crises políticas e sociais regionais e nacionais com implicações internacionais viraram contenciosos que ocorrem ao mesmo tempo e com crescente intensidade ${ }^{9}$. De Paris a Macau a Caracas a Damasco a Lisboa a Nova Iorque a Trípoli a Johanesburgo a Brasília a Kabul a Bagdá, portanto, o "estamos em crise" virou a palavra de ordem de todo cidadão, que em iminente desespero invade ruas ou/e redes sociais demandando de seus mandatários -locais, regionais, mundiais-dias melhores (Badie 2015). Nesse contexto de urgência mundial e dificuldade em definição de prioridades de global governance, milhares de mulheres e homens, em uniforme ou não, vindos dos quatro cantos do planeta, mesmo diante de tanto desconsolo e tanta desilusão, desembarcam em Port-au-Prince desde 2004 com o impulso da MINUSTAH com intuito de conferir auxílio e afeição a essa gente desse país, Haiti, submerso em séculos de desconsolo e desilusão.

Instalada no bojo do traumatismo que a invasão à revelia do Iraque em 2003 causou à legitimidade dos organismos internacionais, a MINUSTAH ainda não chegou a produzir movimentos de transformação profunda nas estruturas de poder e deliberação das Nações Unidas. Segue distante de forjar ambiente para a formulação de um documento estilo An Agenda for Peace do secretário geral Boutros Boutros Ghali (Boutros Ghali 1992; Dejammet 2015; Boutros Ghali 1999). Segue longe de representar um território de inovação e renovação profunda de concepções como o momento das missões das Nações

\footnotetext{
7 S/2004/300 - Report of the Secretary-General on Haiti. United Nations, 16 de abril de 2004.

${ }^{8}$ S/2014/162 - Report of the Secretary-General on the United Nations Stabilization Mission in Haiti. United Nations, 7 de março de 2014.

${ }^{9}$ Crisis Watch, 2016 in http://www.crisisgroup.org/ /media/Files/CrisisWatch/2016/cw149.pdf
} 
Unidas na Namíbia e na América Central veio a representar. Também não pode se comparar com o que se executou, de 1994 a 2000, em missões humanitárias por Angola, Iugoslávia, Ruanda, Bósnia, Somália e Serra Leoa (Berdal 2008 : 175-204). Mesmo assim, ela serve de referência ao Peacebuilding Commission em 2005, cujo objetivo se converte em refletir sobre a ampliação da eficiência das peacekeeping operations, sobretudo na resposta imediatamente "aftermath of conflict"10 e suscita toda sorte de discussão sobre global governnace em tempos de sobreposição de crises.

Desde sua implantação em 1 de junho de 2004, a MINUSTAH permanece objeto de avaliação e discussão internacionais de toda sorte de especialistas. Mas poucos a conhecem mais e melhor que o seu representante legal e moral, a saber, o secretário geral das Nações Unidas. Ao secretário -Kofi Annan no princípio e Ban Ki-moon agora- compete acompanhar e recomendar encaminhamentos. Esse acompanhamento vai rendido ao Conselho de Segurança e à Assembleia Geral na forma de relatórios. Esses relatórios têm como função essencial justificar a necessidade da missão e solicitar a sua continuação como expressão de responsabilidade de governaça. Desde 2004, Kofi Annan e Ban Ki-moon apresentaram dezenas de relatórios a propósito da MINUSTAH. Analisar alguns aspectos desses relatórios, referentes ao período 2004-2014, na tecitura da global governance representa o objetivo central deste artigo.

\section{Preliminares: peacekeeping operations}

Antes de propriamente avançar sobre os relatórios do secretário geral sobre a MINUSTAH, convém discorrer en passant sobre a natureza das peacekeeping operations.

Embora as operações de paz se constituam em prática recorrente das Nações Unidas, criadas a partir de resoluções do Conselho de Segurança (CS) como instrumento de governança, importa ressaltar a necessidade de acordo entre todos os membros permanentes do Conselho para levar a efeito tais resoluções, posto que o voto negativo de apenas um dos membros impossibilita sua aprovação.

Do ponto de vista normativo, tais operações são amparadas nos capítulos VI e VII da Carta das Nações Unidas, que tratam sobre situações conflitivas que ameaçam a paz e a segurança internacionais. O capítulo VII, um dos mais controversos do ponto de vista da história das relações internacionais, possibilita, em seu artigo 42, ao CS "take [...] action by air, sea, or land forces as may be necessary to maintain or restore international peace and security" 11.

${ }_{10} \mathrm{~A} / 63 / 881-\mathrm{S} / 2009 / 304$ - Report of the Secretary-General on peacebuilding in the immediate aftermath of conflict. United Nations, 11 de junho de 2009.

${ }^{11}$ Carta das Nações Unidas. 1945 http://www.un.org/en/documents/charter/chapter7.shtml 
Ainda que não previstas na Carta da Nações Unidas, as operações de manutenção da paz se iniciaram nos primeiros anos da criação da ONU. Em 1948 o Conselho de Segurança autorizou a United Nations Truce Supervision Organization - UNTSO, formada por observadores militares destinados a monitorar o armistício entre israelenses e palestinos. Posteriormente, por ocasião da crise de Suez, em 1956, foi criada a United Nations Emergence Force - UNEF I, a primeira força de paz armada autorizada pela organização. Desde então cada operação criada e enviada a campo se destina às peculiaridades do conflito em questão (Coate; Forsythe; Pease; Weiss 2010).

Em princípio, as peacekeeping operations envolvem os objetivos de apoiar a implementação de acordos de paz ou cessar fogo. A própria Organização ressalta, todavia, que tais operações fazem parte de uma série de atividades direcionadas à manutenção da paz, que devem ocorrer de forma integrada, mas não necessariamente linear, a saber: prevenção e mediação de conflitos, peacemaking, peace enforcement e peacebuilding ${ }^{12}$. Importa notar que, apesar da distinção entre os termos, as hoje chamadas missões robustas, multidimensionais ou complexas podem englobar elementos de todas as categorias elencadas. Cabe, então, um breve retrospecto sobre o desenvolvimento de tais operações, a fim de explicitar a passagem de operações "simples" a "robustas".

Além dos supracitados objetivos primários das peacekeeping operations, destacamos também seus três princípios basilares: consentimento das partes, imparcialidade e não uso da força exceto em legítima defesa. A observação de tais princípios deve contribuir para a legitimação das operações perante a população local, evitando a identificação da ONU como parte no conflito ${ }^{13}$.

Até o final da guerra fria este framework se mostrava adequado ao cumprimento dos objetivos estabelecidos. Entretanto, com a mudança no contexto internacional proporcionada pela queda da URSS, surgiram situações distintas daquelas em que a Organização costumava operar. Se por um lado a menor utilização do poder de veto das potências permanentes possibilitou a aprovação de um maior número de missões e maior margem de ação para a ONU, por outro, os conflitos que eclodiram principalmente nas antigas Repúblicas Soviéticas e na África eram majoritariamente internos, e não mais inter-estatais (Annan 2013; Miall; Ramsbotham; Woodhouse 2005).

12 As primeiras envolvem medidas diplomáticas, dentre as quais destaca-se os bons ofícios do Secretário Geral -tanto para evitar o crescimento das hostilidades entre as partes quanto para negociar acordos-bem como a participação de diversos outros atores como Estados, organizações internacionais regionais e organizações não governamentais. O Peace enforcement se aplica às áreas em conflito identificadas pelo Conselho como ameaças à paz e segurança, e por esse motivo passíveis de medidas coercitivas, inclusive uso de força militar sem o consentimento das partes, quando expressamente autorizado por aquele órgão. Por fim, o peacebuilding tem como objetivo evitar a reincidência de conflitos através do fortalecimento das " national capacities at all levels for conflict management, ando to lay the foundation for sustainable peace and development". UNITED NATIONS. Peace and Security in http://www.un.org/en/peacekeeping/operations/peace.shtml

13 UNITED NATIONS. Peace and Security in http://www.un.org/en/peacekeeping/operations/ peace.shtml

Araucaria. Revista Iberoamericana de Filosofía, Política y Humanidades, año 19, n 37. Primer semestre de 2017. Pp. 353-380. ISSN 1575-6823 e-ISSN 2340-2199 doi: 10.12795/araucaria.2017.i37.17 
Desta forma, evidenciava-se a necessidade de uma abordagem mais completa em sua tratativa, conforme frisado por Boutros-Ghali, então Secretário Geral, em seu An Agenda for Peace - Preventive diplomacy, peacemaking and peace-keeping. De 1992, o Report promoveu um esforço de envergadura em sistematizar e consolidar alguns conceitos atinentes a operações de paz e, obviamente, em discutir sobre o eterno paradigma da soberania. De acordo com o relatório, os direitos humanos e o desenvolvimento social e econômico têm papel crucial na resolução das raízes dos conflitos. Além disso, Boutros - Ghali reforçou ainda a necessidade de integração entre prevenção, peacekeeping, peacemaking e peacebuilding, sinalizando, ainda que a soberania dos Estados pode não ser, necessariamente, absoluta (Boutros Ghali 1992).

Entretanto, as dificuldades de adequação ao novo contexto são explicitadas ao lembrar da década de 1990, marcada por situações trágicas, como a inadequação das operações empreendidas em Somália e Ruanda, e a morosidade na resposta a situações como Bósnia e Kosovo.

Ao assumir o cargo de Secretário Geral, em 1998, uma das prioridades de Kofi Annan foi justamente a elaboração de estratégias visando a melhoria da credibilidade e possibilidades de sucesso das operações. Refletindo este esforço podem ser citados o Relatório Brahimi, o Relatório do Milênio, e o relatório The Responsibility to Protect.

O primeiro resultou do Painel das Nações Unidas Sobre Operações de Paz, realizado no ano 2000 e explicitou a necessidade de uma maior flexibilização na possibilidade de uso da força pelas operações ${ }^{14}$. O segundo, que trouxe as considerações de Annan sobre o papel da ONU no século XXI, teve um capítulo dedicado às diretrizes para a paz e segurança, no qual foi reforçada a importância do fortalecimento das atividades de prevenção de conflitos, com foco nos direitos humanos e desenvolvimento social e econômico. Além disso, defendia que a soberania nacional não poderia ser evocada nos casos de violações dos direitos humanos e ventilava a possibilidade de intervenção armada autorizada pelo Conselho como uma resposta aos casos de assassinatos em massa ${ }^{15}$.

Publicado em 2001, de autoria da Internacional Comission on Intervention and State Sovereignty, o relatório The Responsibility to Protect propunha uma mudança na abordagem direcionada às intervenções humanitárias: estas deveriam ser tratadas a partir do imperativo de proteger as pessoas que sofrem violações de seus direitos humanos. Tal responsabilidade é primária dos Estados, mas a comunidade internacional deve auxiliá-los nesta empreitada,

\footnotetext{
${ }^{14} \mathrm{~A} / 55 / 305$ - Report of the panel on united nations peace operations. 21 de Agosto de 2000 in http://www.un.org/en/ga/search/view_doc.asp?symbol=A/55/305

${ }^{15}$ Kofi Annan, We, the peoples. The Role of United Nations in the 21st century. United Nations, 2000 in http://www.un.org/en/events/pastevents/pdfs/We_The_Peoples.pdf
} 
e agir em defesa das populações, quando estes não o fazem. ${ }^{16}$ Assim, nestes casos, a intervenção armada, mesmo que sem o consentimento das partes, deve ser uma atitude aplicável, legítima e justificada. Apesar de trazer tal inovação quanto ao uso da força, o relatório reforça também a importância da integração entre prevenção, reação e reconstrução, com destaque para a primazia da primeira.

Embora seja um relatório longo e denso, a Responsabilidade de Proteger foi incorporada à ONU apenas nos parágrafos 138 e 139 do documento final da Cúpula Mundial de 2005, que definem os crimes de genocídio, limpeza étnica, crimes de guerra e crimes contra a humanidade como legitimadores da intervenção militar para defesa dos direitos humanos, desde que com a autorização do Conselho. Ademais, reforça também a importância dos esforços preventivos para a manutenção da paz e segurança internacionais ${ }^{17}$.

Além de tais esforços políticos, é digno de menção o esforço de normatizar tais entendimentos acerca das operações de paz em diretrizes internas, com maior possibilidade de aplicação prática, sintetizado na capstone doctrine, documento de alto nível da ONU que define os princípios e guidelines das Peace Operations. Também este documento reforça a importância da abordagem integrada das operações de peacekeeping, e o fato de que dificilmente missões "simples" como as da década de 1950 conseguiriam se adequar ao atual contexto internacional ${ }^{18}$.

\section{Da governaça global}

O fim do conflito Leste-Oeste modificou a densidade e a qualidade de todas as ações no meio internacional. Os Estados Unidos da América acreditaram ter ganhado a guerra fria e decretaram o fim da história. Doravante o mundo inteiro serviria pretensamente aos seus preceitos liberais marinados no american way of life. Mas as fraturas no modelo não tardaram aparecer. Os europeus estavam em vias de consolidar uma nova Europa. Ioguslávia e Iraque demandavam atenção especial dos defensores da nova ordem mundial. Ruanda e Sérvia mostravam a impotência das potências. Os sul-americanos iam se acomodando no Mercosul e em suas novas repúblicas ávidas por democracia. Os russos iam aprendendo a viver depois da URSS. Os chineses e indianos planificavam o seu novo lugar ao sol. Os africanos iam amargando o choque da descolonização.

\footnotetext{
${ }^{16}$ INTERNACIONAL COMISSION ON INTERVENTION AND STATE SOVEREIGNTY. The Responsibility to protect. 2001 in http://responsibilitytoprotect.org/ICISS\%20Report.pdf

17 A/RES/60/1- Documento Final de la Cumbre Mundial 2005. United Nations, 24 de outubro de 2005 in http://www.un.org/es/comun/docs/?symbol=A/RES/60/1

${ }^{18}$ UNITED NATIONS. United Nations Peacekeeping Operations: Principles and Guidelines. 2008 in http://pbpu.unlb.org/pbps/Library/Capstone_Doctrine_ENG.pdf
} 
De súbito, veio o 11 de setembro de 2001 e com ele a revanche de todos aqueles, especialmente muçulmanos, retirados, anteriormente, da história. Não demorou a demonização do Oriente Médio e o apelo à perseguição sem fim do inimigo sem rosto nem nome encarnado no terror. Iraque e Afeganistão voltam às páginas dos jornais. Estados Unidos e seus aliados investiram contra eles. E eis que surgem os BRICs, a quintessência dos países emergentes. Mas Egito, Turquia, Líbia continuavam às voltas com suas tensões por não serem países ricos nem emergentes. Da Eurásia, georgianos e ucranianos reivindicam a soberania nacional de sua integridade territorial enquanto no Cáucaso a demanda segue pelo direito de autodeterminação. Em meio a isso irromperia a crise financeira de 2007-2009. A recomposição de forças vai se impondo. Os $99 \%$ começariam a bradar mais forte contra os do $1 \%$ e da ocupação de Wall Street insuflaram a ocupação da praça Tahrir e de outras praças. A primavera dos povos árabe estava, assim, em marcha. Da Tunísia ao Egito ao Baheim ao Mali à Líbia à Síria a palavra de ordem era modificar seus mandatários. Alguns conseguiram; outros não. Mesmo aos observadores acostumados com as turbulências do mundo contemporâneo, a aceleração dos fenômenos e das crises aflige e constrange (Gaddis 2005; Fontaine 2004 ; Hobsbawm 1995; Kennedy 1987; Fukuyama 1992; Huntington 1996; Badie 2011; Silva 2015; Kissinger 1994). E justamente no bojo dessa aceleração de aceleração de fenômenos, crises e constrangimentos que emergiu -e se estabeleceu- a discussão teórica e prática sobre governança global.

James Rosenau \& Ernst-Otto Czempiel foram dos primeiros a sistematizar a discussão, retirando o foco da "globalização econômica", centrada na dinâmica de mercados, para acentuar a importância da globalidade totalizante e totalizadora dos fenômenos globais do último quartel do século 20 (Rosenau \& Czempiel 1992). Em sua perspectiva, ao menos quatro características globais começavam a emergir após o desaparecimento do bloco soviético. Uma delas era a redefinição das esferas de autoridade onde padrões de integração e fragmentação começaram a interagir em diferentes níveis -individual, local, nacional, regional, global- e nas mais diversas áreas específicas. Outra característica envolvia a emergência de uma sociedade civil global onde fenômenos de mudanças globais permitiriam o estabelecimento de vínculos transnacionais entre organizações não governamentais e da sociedade civil que passam a se organizar em escala global na forma de governança global humana (Falk 1995) ou de uma democracia cosmopolita (Archibugi 1998). A terceira característica era a evidente formação de elites transnacionais na forma de uma classe capitalista global composta por parcelas de elites intelectuais, políticas e econômicas capazes de promover uma agenda liberal no sistema internacional. Por fim, a quarta característica indicava o fortalecimento de comunidades epistêmicas associadas ao conhecimento técnico-científico devido ao 
desenvolvimento e predomínio denovas tecnologias de conhecimento econômico emergente, em especial às tecnologias da chamada revolução informacional (Pereira; Souza; Santos Filho 2011). Essas concepções começaram a ser postas em prática pela The Commission on Global Governance das Nações Unidas, instalada sob demanda do secretário Boutros-Boutros Ghali, em 1994.

Os trabalhos dessa Commission resultaram no documento Our global neighbourhood de 1995. Desse documento emanam os princípios da governança global que estão assentados na convicção de que "There is no alternative to working together and using collective power to create a better world". Pois "Governance is the sum of the many ways individuals and institutions, public and private, manage their common affairs".

Em verdade, essas concepções reafirmam os princípios fundadores das Nações Unidas e os conferem significação moderna ao indicar que, portanto "Effective global decision-making thus needs to build upon and influence decisions taken locally, nationally, and regionally, and to draw on the skills and resources of a diversity of people and institutions at many levels. It must build partnerships -networks of institutions and processes-that enable global actors to pool information, knowledge, and capacities and to develop joint policies and practices on issues of common concern".

A criação e manutenção da MINUSTAH foi -e segue sendo- momento decisivo de aplicação desses conceitos e concepções. O êxito ou fracaso da missão escapa à governança global - e ao escopo deste artigo. Mas é possível afirmar que, diante da situação histórica do Haiti, antes a MINUSTAH que a indiferença. E, a não-indiferença, no caso, foi -e tem sido- a expressão do aprofundamento da discussão teórica e prática da governança global. $\mathrm{Ou}$ seja, a MINUSTAH é um fato. E um fato complexo que demonstra a força e a importância dos aportes da governaça global. O que vai a seguir vai auxiliar a compreensão empírica dessa força.

\section{Ainda jacobinos negros}

The Black Jacobins de C.L.R. James (2000) continua sendo a obra mais decisiva para a compreensão da mutação da colônia mais produtiva das Américas no século 19 no país mais miserável do mundo no século 21. De São Domingos ao Haiti, de Toussaint L'Ouverture ao presidente Jean Bertrand Aristide, agruras sem fim tomaram conta do destino dessa gente ainda sedenta de liberdade.

As eleições de 2000 deram vitória ao presidente Aristide e ao seu partido Fanmi Lavalas. A oposição -composta por partidos políticos e diversos setores da sociedade civil- protestou. Acusou o resultado de forte manipulação. 
Sugeriu a resignação do presidente. Em 2003, o embate político ganhou em proporção e densidade. A comunidade internacional, desassossegada, interveio. A CARICOM, Comunidade do Caribe, foi alçada a mediar. No dia 31 de janeiro de 2004, apresentou o Prior Action Plan cujo objetivo era contemporizar as instabilidades. No sentido mais concreto, sua função era promover "major reforms", incluindo a formação de um novo gabinete ministerial. Para implantar o plano foi criado o Grupo dos Seis, G6, envolvendo Bahamas pela CARICOM, Canadá, União Europeia, França, OEA e Estados Unidos. As discussões avançavam em forma e conteúdo. Tudo era feito para proteger o mandato do presidente Aristide. No entanto, no início de fevereiro de 2004, ocorreu a eclosão de conflitos armados no nordeste do país. A Polícia Nacional do Haiti, havia muito depauperada e submersa em desmando, perdeu totalmente o controle e a legitimidade. Muitos de seus componentes desertaram. Vários deles passaram a compor milícias armadas e grupos paramilitares. Chimères era o mais afamado. Nesse início de fevereiro de 2004, grupos armados, liderados por milicianos e antigos homens da Polícia Nacional, tomaram a cidade de Gonaives e diversas outras no platô central. A politização do conflito armado era evidente. Sua intenção também. E, por isso, os protestos e conflitos não demoraram rumar para Port-au-Prince. Impotente diante da situação, o presidente Aristide acabou por deixar o país. Seguiu ao exílio. Isso ocorreu nas primeiras horas do dia 29 de fevereiro de 2004. Nessas mesmas horas matinais, o primeiro-ministro Yvon Neptune, leu a carta de resignação do presidente Aristide. Nas horas seguintes, Boniface Alexandre, presidente da Suprema Corte, assumiu a presidência interina do Haiti. Mas a tensão do país aumentava em indeterminação. $\mathrm{O}$ histórico de instabilidade social somado à fragilidade política conduzia prognósticos de tempos ainda muito mais difíceis. Por essa razão, o experimentado Boniface Alexandre, agora, presidente interino, de imediato, nas primeiras horas vespertinas, enviou solicitação de assistência às Nações Unidas. Assistência especialmente armada. Essa solicitação foi enviada de pronto ao Conselho de Segurança. O Conselho de Segurança não tardou em acolher o pedido e fazê-lo base da resolução 1529 (2004) ${ }^{19}$.

Essa resolução criou uma força interina multinacional -mutinational interim force, MIF- com duração máxima de três meses. Essa força foi composta por Canadá, Chile, França e Estados Unidos. Seu objetivo era, majoritariamente, estancar a violência e sondar a gravidade da situação para, na sequência, se estabelecer a "follow-on United Nations stabilization force to support continuation of a peaceful and constitutional political process and the maintenance of a secure and stable environment in Haiti" ${ }^{20}$.

${ }_{19} \mathrm{~S} / 2004 / 300$ - Report of the Secretary-General on Haiti. United Nations, 16 de abril de 2004.

${ }_{20} \mathrm{~S} / 2004 / 300$ - Report of the Secretary-General on Haiti. United Nations, 16 de abril de 2004. p. 1. 
Para o primeiro intuito, foram enviados 3.000 soldados dos países concernidos para reestabelecer o controle nas cidades em conturbação. Para o segundo, foi nomeado John Reginald Dumas como special adviser do secretário geral Kofi Annan. Reginald Dumas consultou praticamente todos os envolvidos no processo no Haiti, na OEA, na CARICOM, nos Estados Unidos da América, no Reino Unido e na Jamaica. O resultado dessas consultas, iniciadas no dia 11 de março de 2004, serviu de fundamento para o relatório que selou o destino da MINUSTAH.

Apresentado pelo secretário geral ao Conselho de Segurança no dia 16 de abril de 2004, o relatório recomenda a criação da Mission des Nations Unis pour la stabilization en Haiti e estrutura as normas de seu mandato.

De modo sumário, o secretário geral sugere apoio para:

1. Estruturação do processo constitucional e político

2. Reestabelecimento da tranquilidade e da ordem públicas

3. Processo eleitoral a partir da feitura de eleições democráticas e credíveis.

4. Restauração do rule of law e da seguridade pública

5. Reforma do judiciário e do sistema prisional

6. Bom andamento do governo de transição

7. Boa relação entre o governo de transição e demais grupos políticos

8. Ampliação da legitimidade do governo de transição diante dos governos locais

9. Reconciliação dos diversos grupos

10. Facilidade a provisão, manutenção e captação de assistência humanitária

11. Restauração do serviço público

12. Formulação de estratégia de desenvolvimento sustentável

13. Monitoramento da condição dos direitos humanos

14. Melhor cooperação com agências internacionais de doação

15. Garantia segurança do pessoal envolvido na missão

16. Promoção da participação de mulheres no processo de transição

Essas diretrizes deveriam ser implantadas em vinte e quatro meses, a contar do início da missão, período no qual deveria ser convocado e executado novo processo eleitora ${ }^{21}$.

Essas recomendações foram adotadas pelo Conselho de Segurança no dia 30 de abril e materializadas na resolução 1542 (2004). Essa resolução conferia

${ }^{21}$ S/2004/300 - Report of the Secretary-General on Haiti. United Nations, 16 de abril de 2004. pp. 20-34.

Araucaria. Revista Iberoamericana de Filosofía, Política y Humanidades, año 19, n 37. Primer semestre de 2017. Pp. 353-380. ISSN 1575-6823 e-ISSN 2340-2199 doi: 10.12795/araucaria.2017.i37.17 
mandato de seis meses à missão que teria início no dia $1^{\circ}$ de junho de 2004. Durante todo o mês de maio de 2004, a MINUSTAH foi se fazendo e a Força Interina Multinacional, se desintegrando. Para responsável pela representação especial do Haiti e da missão fora indicado o chileno Juan Gabriel Valdés. Para representante da coordenação de assuntos humanitários e de desenvolvimento, Adama Guindo. Para o comando das forças militares, o general brasileiro Augusto Heleno Ribeiro Pereira. Para das forças policiais, o canadense David Charles Beer ${ }^{22}$.

\section{O peso do número}

De 18 de novembro de 2004 a 7 de março de 2014, o secretário geral das Nações Unidas apresentou vinte relatórios ao Conselho de Segurança. O objetivo essencial dos relatórios foi sempre descrever o substantivo das atividades desenvolvidas pela missão durante o período do mandato. Os relatórios -componentes da liturgia do processo decisório no interior das Nações Unidas para além da tensão entre a Assembleia Geral e o Conselho de Segurança (Zaum 2008:155-174) - são onde o secretário geral rende contas da missão. Contas rendidas, o relatório também serve de fundamento para solicitação de renovação do mandato. De $1^{\circ}$ de junho de 2004 a 7 de março de 2014 foram aprovadas doze resoluções -1608 (2005), 1702 (2006), 1743 (2007), 1780 (2007), 1840 (2008), 1892 (2009), 1908 (2010), 1927 (2010), 1944 (2010), 2012 (2011), 2070 (2012) e 2119 (2013)- de renovação da MINUSTAH ${ }^{23}$.

De maneira geral, os relatórios vão estruturados sobre os eixos principais da missão. Primeiro ponto corresponde à segurança. $\mathrm{O}$ segundo, à dimensão política. O terceiro, ao respeito aos direitos humanos. O quarto, aos esforços na promoção de desenvolvimento econômico, social e humanitário. O quinto transcreve as necessidades que ainda precisam ser supridas para conferir a estabilização do país.

De relatório em relatório se vai descobrindo a complexidade da situação do Haiti e a concomitante complexidade das operações internacionais de melhoramento do Haiti.

O Haiti era em 2004 - e continua sendo em 2014-um dos países mais pobres do Ocidente. Seu setor comercial era frágil, decadente e/ou inexistente. $40 \%$ da liquidez do meio circulante advinha dos envios de dinheiro de familiares de haitianos vivendo no exterior. $52 \%$ da população vivia com menos de 1,5 dólar

${ }^{22}$ S/2004/698 - Interim Report of the Secretary-General on the United Nations Stabilization Mission in Haiti. United Nations, 30 de agosto de 2004.

${ }^{23}$ S/2014/162 - Report of the Secretary-General on the United Nations Stabilizations Mission in Haiti. United Nations, 7 de março de 2014.

Araucaria. Revista Iberoamericana de Filosofía, Politica y Humanidades, año 19, n 37. Primer semestre de 2017. Pp. 353-380. ISSN 1575-6823 e-ISSN 2340-2199 doi: 10.12795/araucaria.2017.i37.17 
ao dia. As mulheres estavam submetidas a condições nefandas de violência doméstica e precariedade humanitária. Parte substantiva delas não dispunha de educação reprodutiva. Parte importante dessa parte substantiva era sujeita a altos índices de mortalidade no parto. Consequentemente, a mortalidade infantil também era imensa. Uma das maiores do mundo. Apenas 34\% das crianças de 12 a 23 meses tinham recebido alguma vacina nos anos que antecederam a MINUSTAH. 16\% não receberam vacina alguma. O resto do percentual para completar $100 \%$, morrera antes dos 12 meses de vida. $51 \%$ dos 8 milhões e meio de habitantes do Haiti dos tempos do presidente Aristide estavam submersos em desnutrição crônica. 2/3 das crianças com menos de cinco anos sofriam de anemia. $40 \%$ das meninas, crianças e adolescentes, jamais acessaram a escola primária. Mais de duas mil, em média por ano, crianças e adolescentes eram vendidas no black market internacional de pessoas. $75 \%$ de todas as crianças entre 7 e 14 anos eram objeto de alguma exploração, de sexual a moral. 5\% das moças e $4,1 \%$ dos moços entre 14 e 24 anos eram portadores de HIV/AIDS. $4,5 \%$ de toda a população eram portadores do vírus, pelas estimativas de 2002 . 30 mil pessoas morreram em média por ano de 1980 até 2004 por conta desse vírus. Mais de 200 mil crianças, nesse mesmo conjunto de razão, eram deixadas órfãs ${ }^{24}$.

Esse retrato, com a adição de outras múltiplas cores, justificava o imperativo da necessidade da missão antes, durante e depois da saída do presidente Aristide.

\section{Morrer pelo Haiti}

O vazio de poder tornou mais aguda a combinação perversa e malfazeja desses números. Os dez anos de MINUSTAH não necessariamente reverteram esses dados. Mas seu esforço foi sem fim.

A primeira leva de pés estrangeiros para estabilização do Haiti era composta de 2.755 soldados e oficiais de doze países -Argentina, Benin, Bolívia, Brasil, Chile, Croácia, França, Nepal, Paraguai, Peru, Estados Unidos da América e Uruguai-e 365 policiais de 17 países -Benin, Bósnia e Herzergovina, Burkina Faso, Camarões, Canadá, Chad, Chile, China, França, Ghana, Jordânia, Mali, Nigéria, Portugal, Senegal Serra Leoa e Turquia (ver tabela 1 e tabela 2)

${ }^{24}$ S/2004/300 - Report of the Secretary-General on Haiti. United Nations, 16 de abril de 2004. pp. 12-13. 
Tabela 1

\begin{tabular}{|l|c|c|c|}
\hline \multicolumn{4}{|c|}{$\begin{array}{c}\text { Contingente de militares da MINUSTAH } \\
\text { 17e agosto de 2004 }\end{array}$} \\
\hline País & Oficiais & Soldados & Total \\
\hline Argentina & 6 & 480 & 486 \\
\hline Benin & 3 & - & 3 \\
\hline Bolívia & 6 & - & 6 \\
\hline Brasil & 12 & 1.198 & 1.210 \\
\hline Chile & 6 & 448 & 454 \\
\hline Croácia & 1 & - & 1 \\
\hline França & 2 & - & 2 \\
\hline Nepal & 6 & - & 6 \\
\hline Paraguai & 6 & - & 6 \\
\hline Peru & 2 & - & 2 \\
\hline Estados Unidos da América & 4 & - & 4 \\
\hline Uruguai & 4 & 571 & 575 \\
\hline Total & 58 & 2.697 & 2.755 \\
\hline Font S-2004-698, p. & & &
\end{tabular}

Fonte: S-2004-698, p. 16.

Tabela 2

\begin{tabular}{|l|c|c|c|}
\hline \multicolumn{5}{|c|}{$\begin{array}{c}\text { Contingente de policiais e civis da MINUSTAH } \\
17 \text { de agosto de } 2004\end{array}$} \\
\hline \multicolumn{1}{|c|}{ País } & Oficiais civis & Policiais & Total \\
\hline Benin & 9 & - & 9 \\
\hline Bosnia e Hezergovina & 3 & - & 3 \\
\hline Burkina Faso & 39 & - & 39 \\
\hline Camarões & 43 & - & 43 \\
\hline Canadá & 26 & - & 26 \\
\hline Chad & 1 & - & 1 \\
\hline Chile & 2 & - & 2 \\
\hline China & 1 & - & 1 \\
\hline França & 32 & - & 32 \\
\hline Gana & 2 & - & 2 \\
\hline Jordânia & 1 & 125 & 126 \\
\hline
\end{tabular}




\begin{tabular}{|l|c|c|c|}
\hline Mali & 8 & - & 8 \\
\hline Nigéria & 7 & - & 7 \\
\hline Portugal & 5 & - & 5 \\
\hline Senegal & 51 & - & 51 \\
\hline Serra Leoa & 5 & - & 5 \\
\hline Turquia & 5 & - & 5 \\
\hline Total & 240 & 125 & 365 \\
\hline
\end{tabular}

Fonte: S-2004-698, p. 16

O relatório de março de 2014, o mais recente em análise, apresenta a dimensão da evolução do esforço internacional. Nesses dez anos, a quantidade de soldados mais que dobrou, passou dos iniciais 2.755 para 5.814 e o número de 365 policiais foi multiplicado por quase sete vezes e passou para 2.411 (ver tabela 3 e tabela 4 )

Tabela 3

\begin{tabular}{|l|c|c|c|}
\hline \multicolumn{5}{|c|}{$\begin{array}{c}\text { Contingente de militares da MINUSTAH } \\
\text { 10 de fevereiro de 2014 }\end{array}$} \\
\hline País & Oficiais & Soldados & Total \\
\hline Argentina & 11 & 561 & 572 \\
\hline Bolívia & 3 & 205 & 208 \\
\hline Brasil & 18 & 1416 & 1434 \\
\hline Canadá & 5 & 2 & 7 \\
\hline Chile & 3 & 454 & 457 \\
\hline El Salvador & 1 & 34 & 35 \\
\hline Equador & 1 & 52 & 53 \\
\hline França & 2 & - & 2 \\
\hline Guatemala & 2 & 133 & 135 \\
\hline Honduras & & 1 & 1 \\
\hline Indonésia & 1 & 167 & 168 \\
\hline Jordânia & 10 & 241 & 251 \\
\hline Nepal & 13 & 21 & 34 \\
\hline Paraguai & 3 & 114 & 117 \\
\hline Peru & 6 & 367 & 373 \\
\hline Filipinas & 2 & 157 & 159 \\
\hline
\end{tabular}




\begin{tabular}{|l|c|c|c|}
\hline Coréia do Sul & 2 & - & 2 \\
\hline Sri Lanka & 11 & 850 & 861 \\
\hline $\begin{array}{l}\text { Estados Unidos } \\
\text { da América }\end{array}$ & 8 & - & 8 \\
\hline Uruguai & 10 & 927 & 937 \\
\hline Total & 112 & 5.702 & 5.814 \\
\hline
\end{tabular}

Fonte: S-2014-162, p. 20.

Tabela 4

\begin{tabular}{|c|c|c|c|}
\hline \multicolumn{4}{|c|}{$\begin{array}{c}\text { Contingente de policiais e civis da MINUSTAH } \\
10 \text { de fevereiro de } 2014\end{array}$} \\
\hline País & Oficiais civis & Policiais & Total \\
\hline Argentina & 12 & - & 12 \\
\hline Bangladesh & 5 & 216 & 221 \\
\hline Benin & 41 & - & 41 \\
\hline Brasil & 10 & - & 10 \\
\hline Burkina Faso & 55 & - & 55 \\
\hline Burundi & 43 & - & 43 \\
\hline Camarões & 36 & - & 36 \\
\hline Canadá & 81 & - & 81 \\
\hline África Central & 1 & - & 1 \\
\hline Chad & 5 & - & 5 \\
\hline Chile & 14 & - & 14 \\
\hline Colômbia & 27 & - & 27 \\
\hline Costa do Marfim & 132 & - & 132 \\
\hline Croácia & 5 & - & 5 \\
\hline Egito & 16 & - & 16 \\
\hline França & 22 & - & 22 \\
\hline Granada & 2 & - & 2 \\
\hline Guiné & 15 & - & 15 \\
\hline Índia & 5 & 420 & 425 \\
\hline Jordânia & 11 & 278 & 289 \\
\hline Quirsquistão & 1 & - & 1 \\
\hline Lituânia & 1 & - & 1 \\
\hline
\end{tabular}




\begin{tabular}{|l|c|c|c|}
\hline Mali & 37 & - & 37 \\
\hline Nepal & 2 & 140 & 142 \\
\hline Niger & 51 & - & 51 \\
\hline Nigéria & 3 & - & 3 \\
\hline Noruega & 6 & - & 6 \\
\hline Paquistão & - & 139 & 139 \\
\hline Paraguai & 1 & - & 1 \\
\hline Filipinas & 13 & - & 13 \\
\hline Portugal & 1 & - & 1 \\
\hline România & 23 & - & 23 \\
\hline Rússia & 9 & - & 9 \\
\hline Ruanda & 13 & 140 & 153 \\
\hline Senegal & 6 & 140 & 146 \\
\hline Espanha & 9 & - & 9 \\
\hline Sri Lanka & 8 & - & 8 \\
\hline Tunísia & 14 & - & 14 \\
\hline Turquia & 12 & - & 12 \\
\hline Tailândia & 7 & - & 7 \\
\hline Reino Unido & 1 & - & 1 \\
\hline Estados Unidos & 60 & - & 60 \\
\hline Uruguai & 4 & - & 4 \\
\hline Vannuatu & 2 & - & 2.411 \\
\hline Yemem & 18 & - & \\
\hline Total & 838 & 1.573 & \\
\hline Font: S-2014-162, pp & $21-22$ & & 18 \\
\hline
\end{tabular}

Fonte: S-2014-162, pp. 21-22

A simples observação das cartas de operação de 2004 e 2014 amplia a ilustração dessa evolução. 
Mapa 1: Minusth 2004

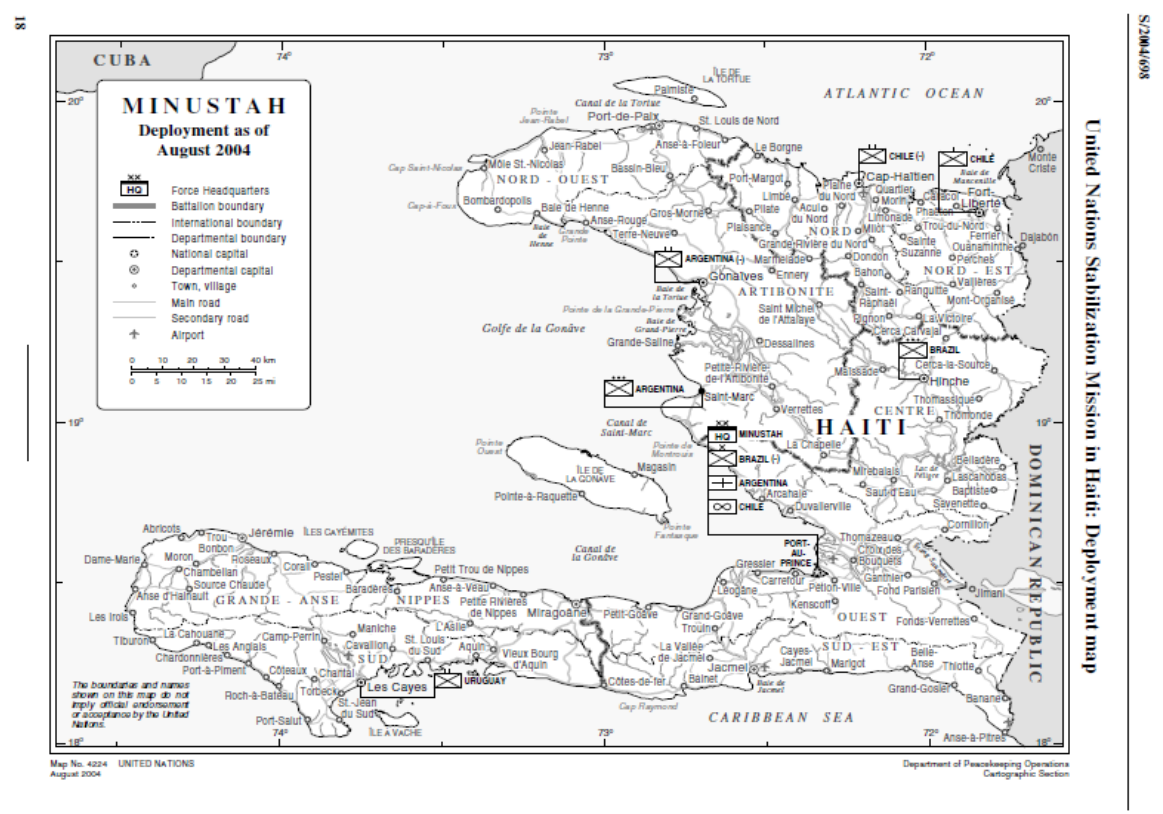

Mapa 2: Minusth em 2014

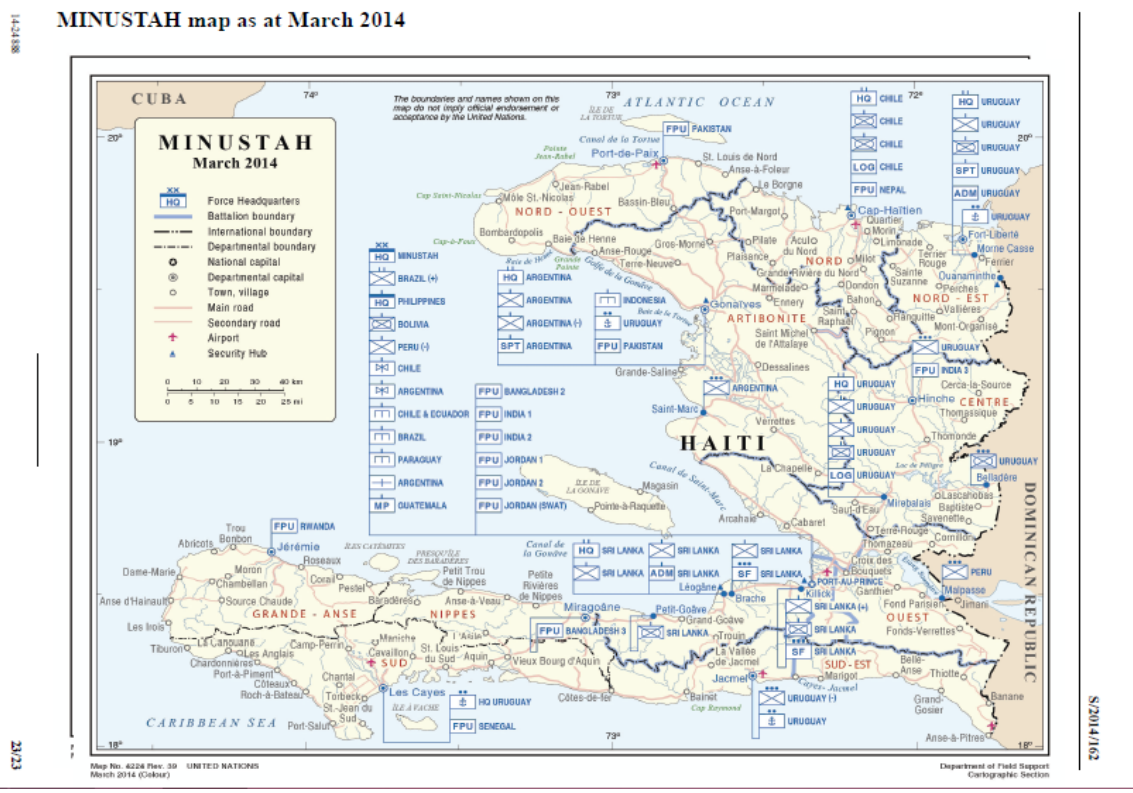

Araucaria. Revista Iberoamericana de Filosofía, Política y Humanidades, año 19, nº 37. Primer semestre de 2017. Pp. 353-380. ISSN 1575-6823 e-ISSN 2340-2199 doi: 10.12795/araucaria.2017.i37.17 
A abordagem direta do primeiro e do último relatórios isolados esconde diversos segredos internos do período. Diferente do que se poderia imaginar pela simples avaliação dos números deles em exclusivo, a quantidade de soldados e policiais avança progressivamente de um mandato ao outro (ver tabela 5). Dos 2.755 soldados inicias, chega a 7.519 em começos de 2006 e a 8.728 em agosto de 2011. Dos 365 policiais de 2004 vai num crescendo até chegar aos 3.447 em agosto de 2011. Após agosto de 2011, a quantidade de militares e policiais vai diminuindo até aos atuais 5.814 e 2.411 (fevereiro de 2014).

Tabela 5

\begin{tabular}{|c|c|c|c|}
\hline \multicolumn{4}{|c|}{ Evolução da quantidade de militares e policiais } \\
\hline 17 de agosto de 2004 & Militares & Policiais & Totais \\
\hline 8 de novembro de 2004 & 2.755 & 365 & 3.120 \\
\hline 15 de fevereiro de 2005 & 6.493 & 978 & 5.471 \\
\hline 9 de maio de 2005 & 6.211 & 1.398 & 7.411 \\
\hline 29 de setembro de 2005 & 6.381 & 1.471 & 7.852 \\
\hline 31 de janeiro de 2006 & 7.519 & 1.777 & 9.296 \\
\hline 15 de julho de 2006 & 6.309 & 1.687 & 7.996 \\
\hline 10 de dezembro de 2006 & 6.662 & 1.742 & 8.404 \\
\hline 15 de agosto de 2007 & 7.080 & 1.770 & 8.850 \\
\hline 26 de março de 2008 & 7.065 & 1.937 & 9.002 \\
\hline 18 de agosto de 2008 & 6.952 & 1.925 & 8.877 \\
\hline 26 de fevereiro de 2009 & 7.032 & 2.021 & 9.053 \\
\hline 25 de agosto de 2009 & 7.058 & 2.052 & 9.110 \\
\hline 13 de abril de 2010 & 8.306 & 2.177 & 10.483 \\
\hline 23 de agosto de 2010 & 8.548 & 3.063 & 11.611 \\
\hline 23 de agosto de 2011 & 8.728 & 3.447 & 12.175 \\
\hline 15 de agosto de 2012 & 7.272 & 2.807 & 10.079 \\
\hline 31 de julho de 2013 & 6.198 & 2.522 & 8.720 \\
\hline 10 de fevereiro de 2014 & 5.814 & 2.411 & 8.225 \\
\hline Font $2004 / 698$, pp. 16-17; $/ 2004 / 908$, & $14-16 ; \mathrm{S} / 2005$ & 124 \\
\hline
\end{tabular}

Fonte: S/2004/698, pp. 16-17; S/2004/908, pp. 14-16; S/2005/124, pp. 15-17; S/2005/313, pp. 16-18; S/2005/631, pp. 14-16; S/2006/60, pp. 17-19; S/2006/592, pp. 21-23; S/2006/1003, pp. 16-18; S/2007/503, pp. 19-21; S/2008/202, pp. 18-20; S/2008/586, pp. 26-28; S/2009/129, pp. 20-22; S/2009/439, pp. 25-27; S/2010/200, pp. 22-24; S/2010/446, pp. 19-21; S/2011/540, pp.16-18; S/2012/678, pp. 18-20; S/2013/493, pp. 19-21; S/2014/162, pp. 20-22. 
O relatório de agosto de 2011, que traz esses números da quantidade máxima de militares e policiais internacionais empregados no Haiti, cobre, em verdade os dois períodos imediatamente após o devastador terremoto de 12 de janeiro de 2010.

O terremoto e seus efeitos colaterais apresentam dados e conjunturas macabras e desoladoras. Sete pontos na escala Richter foi a sua magnitude: Port-au-Prince, Petit Goâve, Grande Gâve, Léogâne, cidade de Jacmel e cidade de Miragâne foram os principais núcleos populacionais atingidos. $222 \mathrm{mil} \mathrm{e}$ 570 pessoas foram mortas. Dezenas de milhares foram lesionadas. Mais de 1 milhão e meio ficou desabrigado. Outro quase 2 milhões passou a vagar pelo interior do país e do continente. 77 oficiais da Polícia Nacional foram mortos. Centenas deles foram também lesionados. Mais de 4 mil dos 8 mil 535 presos escaparam das prisões. Centenas deles passaram a liderar gangues e grupos de malfeitores por todo o Haiti. Nem mesmo os hóspedes do Hotel Christopher, os oficiais das Nações Unidas, passaram ilesos ao terremoto: 101 deles perderam suas vidas ${ }^{25}$.

Por impulso do momento, a quantidade de soldados passou de 7.058 a 8.306 e a de policiais de 2.052 a 2.177 (ver tabela 6). No caso dos soldados, sim, foi expressivo o acréscimo em função do terremoto. Ocorreu uma agudização da tendência. Mesmo efeito não se notou no caso do acréscimo de policiais que praticamente seguiu uma evolução média padrão.

Tabela 6

\begin{tabular}{|l|c|c|c|}
\hline \multicolumn{4}{|c|}{ Contingente militar da MINUSTAH após o terremoto de 2010 } \\
\hline & Oficiais & Soldados & Totais \\
\hline Argentina & 9 & 551 & 560 \\
\hline Bolívia & 3 & 205 & 208 \\
\hline Brasil & 22 & 2.166 & 2188 \\
\hline Canadá & 11 & & 11 \\
\hline Chile & 5 & 498 & 503 \\
\hline Equador & 2 & 66 & 66 \\
\hline França & 4 & & 2 \\
\hline Guatemala & 1 & 143 & 147 \\
\hline Índia & 2 & & 1 \\
\hline Japão & 8 & 190 & 192 \\
\hline Jordânia & \multicolumn{3}{|c|}{} \\
\hline
\end{tabular}

${ }^{25} \mathrm{~S} / 2010 / 200$ - Report of the Secretary-General on the United Nations Stabilization Mission in Haiti. United Nations, 22 de fevereiro de 2010. pp. 1-3. 


\begin{tabular}{|l|c|c|c|}
\hline Nepal & 13 & 1.067 & 1.080 \\
\hline Paraguai & & 31 & 31 \\
\hline Peru & 5 & 214 & 219 \\
\hline Filipinas & 2 & 155 & 157 \\
\hline Coréia do Sul & 1 & 239 & 240 \\
\hline Sri Lanka & 8 & & 8 \\
\hline $\begin{array}{l}\text { Estados Unidos } \\
\text { da América }\end{array}$ & 8 & & 8 \\
\hline Uruguai & 13 & 1.176 & 1.189 \\
\hline Total & 120 & 8.186 & 8.306 \\
\hline
\end{tabular}

Fonte: S/2010/200, p. 22.

O caso do Brasil, no que tange ao número de envio de soldados, parece ser sintomático. Como comandante militar da missão, o Brasil acaba sendo o país que mais decididamente amplia o seu contingente de homens no Haiti após o terremoto. Faz saltar dos 1.260 homens de agosto de 2009 para 2.160 em abril de 2010, imediatamente após o terremoto. 1.000 soldados a mais (ver tabela 7).

Tabela 7

\begin{tabular}{|c|c|c|c|c|c|}
\hline \multicolumn{6}{|c|}{ Militares brasileiros no Haiti } \\
\hline & \multicolumn{2}{|c|}{ Oficiais } & \multicolumn{2}{|c|}{ Soldados } & Totais \\
\hline 17 de agosto de 2004 & \multicolumn{2}{|c|}{12} & \multicolumn{2}{|c|}{1.198} & 1.210 \\
\hline 8 de novembro de 2004 & \multicolumn{2}{|c|}{12} & \multicolumn{2}{|c|}{1.197} & 1.209 \\
\hline 15 de fevereiro de 2005 & \multicolumn{2}{|c|}{12} & \multicolumn{2}{|c|}{1.200} & 1.212 \\
\hline 9 de maio de 2005 & \multicolumn{2}{|c|}{12} & \multicolumn{2}{|c|}{1.200} & 1.212 \\
\hline 29 de setembro de 2005 & \multicolumn{2}{|c|}{20} & \multicolumn{2}{|c|}{1.199} & 1.219 \\
\hline 31 de janeiro de 2006 & \multicolumn{2}{|c|}{22} & \multicolumn{2}{|c|}{1.200} & 1.222 \\
\hline 15 de julho de 2006 & \multicolumn{2}{|c|}{14} & \multicolumn{2}{|c|}{1.200} & 1.214 \\
\hline 10 de dezembro de 2006 & \multicolumn{2}{|c|}{13} & \multicolumn{2}{|c|}{1.198} & 1.211 \\
\hline \multirow[t]{2}{*}{15 de agosto de 2007} & \multicolumn{2}{|c|}{15} & \multicolumn{2}{|c|}{1.196} & 1.211 \\
\hline & Feminino & Masculino & Feminino & Masculino & \\
\hline 26 de março de 2008 & & 14 & 6 & 1.193 & 1.213 \\
\hline 18 de agosto de 2008 & & 14 & 6 & 1.193 & 1.213 \\
\hline 26 de fevereiro de 2009 & & 15 & 6 & 1.261 & 1.282 \\
\hline 25 de agosto de 2009 & & 15 & 7 & 1.260 & 1.282 \\
\hline
\end{tabular}




\begin{tabular}{|l|l|l|l|l|l|}
\hline 13 de abril de 2010 & & 22 & 6 & 2.160 & 2.188 \\
\hline 23 de agosto de 2010 & & 23 & 9 & 2.133 & 2.165 \\
\hline 23 de agosto de 2011 & & 22 & 17 & 2.146 & 2.186 \\
\hline 15 de agosto de 2012 & & 19 & 21 & 1.856 & 1.896 \\
\hline 31 de julho de 2013 & 18 & 16 & 1.369 & 1.403 \\
\hline 10 de fevereiro de 2014 & & 18 & 14 & 1.402 & 1.434 \\
\hline
\end{tabular}

Fonte: S/2004/698, p. 16; S/2004/908, p. 14; S/2005/124, p. 15; S/2005/313, p. 16; S/2005/631, p. 14; S/2006/60, p. 17; S/2006/592, p. 21; S/2006/1003, p. 16; S/2007/503, p. 19; S/2008/202, p. 18; S/2008/586, p. 26; S/2009/129, p. 20; S/2009/439, p. 25; S/2010/200, p. 22; S/2010/446, p. 19; S/2011/540, p.16; S/2012/678, p. 18; S/2013/493, p. 19; S/2014/162, p. 20.

Essa afirmação sobre o Brasil - que não deixa de ser correta- ficaria muito incompleta sem se levar em conta a first responder do Canadá e dos Estados Unidos que, sós, nas primeiras 72 horas, fizeram desembarcar em Port-auPrince 18 mil e 500 soldados americanos e 2 mil soldados canadenses ${ }^{26}$.

\section{Além do secretário}

Após enviar seus mais de 18 mil homens ao Haiti, o presidente Obama reafirmou em importante artigo saído na Newsweek de 22 de fevereiro de 2010 que "that is who we are. That is what we do" como demonstração da excepcionalidade americana. Nos meses que se seguiram, esse argumento foi recorrente nas manifestações do presidente americano e chegou ao momento mais intenso de concretude diante dos lordes do parlamento inglês na denominação de que

Countries like China, India, and Brazil are growing by leaps and bounds. We should welcome this development, for it has lifted hundreds of millions from poverty around the globe, and created new markets and opportunities for our own nations. And yet, as this rapid change has taken place, it's become fashionable in some quarters to question whether the rise of these nations will accompany the decline of American and European influence around the world. Perhaps, the argument goes, these nations represent the future, and the time for our leadership has passed. That argument is wrong. The time for our leadership is now ${ }^{27}$.

${ }^{26}$ S/2010/200 - Report of the Secretary-General on the United Nations Stabilization Mission in Haiti. United Nations, 22 de fevereiro de 2010. p. 2.

27 Remarks by the President to Parliament in London, United Kingdom, May 25, 2011. 
Em realce aos tempos de ilusão de superpotência isolada dos momentos após o desaparecimento da URSS, os Estados Unidos do presidente Obama aproveita a repercussão internacional de sua atuação na resposta humanitária ao terremoto no Haiti para realinhar a discussão sobre eventual importância da ascensão de alguns países emergentes aglutinados no espaço BRICs. Esses países tiveram ampliação de sua visibilidade nos momentos iniciais da crise financeira e, sobretudo, nas reuniões do G20 em Washington e Londres. Do setembro de 2008, quebra do Lehman Brothers em Nova Iorque, ao janeiro de 2010 no Haiti, os Estados Unidos pareciam ter dado adeus às eras do presidente Bush de ativismo internacional. O presidente Obama, no caso do Haiti, afirma que certamente não. Mas quem olha os números da participação americana na MINUSTAH se depara a imenso contraste (ver tabela 8).

Tabela 8

\begin{tabular}{|c|c|c|c|}
\hline \multicolumn{4}{|c|}{ Militares americanos na MINUSTAH } \\
\hline & Oficiais & Soldados & Totais \\
\hline 17 de agosto de 2004 & 4 & & 4 \\
\hline 8 de novembro de 2004 & 4 & & 4 \\
\hline 15 de fevereiro de 2005 & 4 & & 4 \\
\hline 9 de maio de 2005 & 4 & & 4 \\
\hline 29 de setembro de 2005 & 4 & & 4 \\
\hline 31 de janeiro de 2006 & 4 & & 4 \\
\hline 15 de julho de 2006 & 4 & & 4 \\
\hline 10 de dezembro de 2006 & 3 & & 3 \\
\hline 15 de agosto de 2007 & 3 & & 3 \\
\hline 26 de março de 2008 & 3 & & 3 \\
\hline 18 de agosto de 2008 & 3 & & 3 \\
\hline 26 de fevereiro de 2009 & 4 & & 4 \\
\hline 25 de agosto de 2009 & 3 & & 3 \\
\hline 13 de abril de 2010 & 7 & & 7 \\
\hline 23 de agosto de 2010 & 2 & & 2 \\
\hline 23 de agosto de 2011 & 8 & & 8 \\
\hline 15 de agosto de 2012 & 9 & & 9 \\
\hline 31 de julho de 2013 & 9 & & 9 \\
\hline 10 de fevereiro de 2014 & 8 & & 6 \\
\hline
\end{tabular}

Fonte: S/2004/698, p. 16; S/2004/908, p. 14; S/2005/124, p. 15; S/2005/313, p. $16 ; \mathrm{S} / 2005 / 631$, p. $14 ; \mathrm{S} / 2006 / 60$, p. 17 ; S/2006/592, p. 21; S/2006/1003, p. 16; S/2007/503, p. 19; S/2008/202, p. 18; S/2008/586, p. 26; S/2009/129, p. 
20; S/2009/439, p. 25; S/2010/200, p. 22; S/2010/446, p. 19; S/2011/540, p.16; S/2012/678, p. 18; S/2013/493, p. 19; S/2014/162, p. 20.

Para a MINUSTAH, os Estados Unidos praticamente não enviou soldados. Seu envolvimento contou apenas com oficiais. Por outro lado, sua ajuda unilateral da Joint Task Force Haiti (JTF-Haiti) representou o maior esforço militar da história do país em Disaster Relief. No auge de seus esforços a Operation Unified Response, coordenada pela JTF-Haiti, estando sob a guarda do Comando Sul dos Estados Unidos da América (US Southern Command SOUTHCOM), mobilizou 22 mil pessoas, 33 navios da frota estadunidense e mais de 300 aviões (Cecchine ; Morgan 2013).

Quais, então, as razões que explicam a ausência dos EUA, assim como a França e outros membros permanentes do Conselho de Segurança nos esforços empregados pela MINUSTAH? Sobre essa discussão nada consta nos relatórios do Secretário Geral.

Mas constam nos relatórios -como em alguns rápidos aspectos se procurou apresentar neste artigo-as razões que conduziram, e ainda conduzem, muitos membros da comunidade internacional a apoiar o ideal que remonta aos pais fundadores do país dos tempos Toussaint L'Ouverture, que almejavam simplesmente deixar a pátria livre ou morrer pelo Haiti. 


\section{Referências}

A/55/305 - Report of the panel on united nations peace operations. 21 de Agosto de 2000 in http://www.un.org/en/ga/search/view_doc. asp?symbol=A/55/305

A/59/2005 - In Larger Freedom: Towards Security, Development and Human Rigths for All - Report of the Secretary-General. United Nations, 2 de março de 2005. Parágrafo 154.

A/63/881-S/2009/304 - Report of the Secretary-General on peacebuilding in the immediate aftermath of conflict. United Nations, 11 de junho de 2009. A/RES/60/1- Documento Final de la Cumbre Mundial 2005. United Nations, 24 de outubro de 2005 in http://www.un.org/es/comun/docs/?symbol=A/ $\mathrm{RES} / 60 / 1$

Annan, Kofi, We, the peoples. The Role of United Nations in the 21st century. United Nations, 2000 in http://www.un.org/en/events/pastevents/pdfs/ We_The_Peoples.pdf

Annan, Kofi, Invervenções: Uma vida de guerra e paz, São Paulo, Companhia das Letras, 2013.

Archibugi, Daniele, et. Al, Re-imagining political community: studies in cosmopolitan democracy, Stanford, Stanford University Press, 1998.

Badie, Bertrand, La diplomatie de connivence: les dérives oligarchiques du système international, Paris, Editions la couverte, 2011.

Badie, Bertrand, Le temps des humiliés : pathologie des relations internationales, Paris, Odile Jacob, 2014.

Badie, Bertrand, Un monde de souffrances. Ambivalence de la mondialisation, Paris, Salvator, 2015.

Berdal, Mats, The Security Council and peacekeeping in Vaughan Lowe; Adam Roberts; Welsh, Jennifer; Zaum, Dominik, The United Nations Security Council and War: the evolution of thought and practice since 1945, New York, Oxford University Press, 2008, págs. 175-204.

Boutros Ghali, Boutros, An agenda for Peace, United Nations, 1992.

Boutros Ghali, Boutros, Unvanquished - a U.S.-U.N. saga, New York, Random House, 1999.

Cecchine Gary; Morgan, Forrest, et. All, The U.S. Military Response to the 2010 Haiti Eartquake - Considerations for Army Leaders (RAND Corporation, 2013) in http://www.rand.org/content/dam/rand/pubs/research_reports/ RR300/RR304/RAND_RR304.pdf

Coate, Roger; Forsythe, David; Pease, Kelly-Kate; Weiss, Thomas, The United Nations and Changing World Politics, Boulder, Westview Press, 2010.

Crisis Watch, 2016 in http://www.crisisgroup.org/ /media/Files/ CrisisWatch/2016/cw149.pdf 
Cunliffe, Philip, The Politics of Global Governance in UN Peacekeeping, "International Peacekeeping" 16:3, (2009) págs 323-336.

da Silva, Daniel Afonso, Após Geronimo, "Política Externa (USP)", v. 22 (2014), págs. 109-122.

da Silva, Daniel Afonso, De Bengazhi, uma flor, "Política Externa (USP)" vol. 23 (2015), págs 100-120, 2015.

Dejammet, Alain, Boutros Boutros-Ghali: Une histoire égyptiene, Paris, Erick Bonnier, 2015

Dejammet, Alain, L'incendie planétaire. Que fait l'ONU?, Paris, Cerf, 2015.

Falk, Richard, On humane governance: toward a new global politics, Pennsylvania, Pennsylvania State University Press, 1995.

Ferguson, Niall, The great degeneration, New York, Random House, 2013.

Fontaine, André, La guerre froide, 1917-1991, Paris: la martinière, 2004.

Fontana I Làzaro, Josep, El futuro es un país estraño. Barcelona: Passado \& Presente, 2013.

Fontana I Làzaro, Josep, Por el bien del imperio: una historia del mundo desde 1945, Barcelona, Passado \& Presente, 2011.

Fukuyama, Francis, Political order and political decay: from the Industrial revolution to the globalization of democracy, London, Profile Books, 2014.

Fukuyama, Francis, The End of History and the Last Man, New York, Free press, 1992.

Gaddis, John Lewis, The cold war: a new history, New York, Penguin, 2005.

Hobsbawm, Eric J., The Age of Extremes: A History of the World, 1914-1991, London, verso, 1995.

Huntington, Samuel P., The Clash of Civilizations and the Remaking of World Order, New York: Simon \& Schuster, 1996.

INTERNACIONAL COMISSION ON INTERVENTION AND STATE SOVEREIGNTY. The Responsibility to protect. 2001 in http:// responsibilitytoprotect.org/ICISS\%20Report.pdf

James, C. L. R, Os jacobinos negros. Toussaint L'Ouverture e a revolução de São Domingos, São Paulo, Boitempo, 2000.

Kennedy, Paul, The Rise and Fall of the Great Powers: Economic Change and Military Conflict From 1500 to 2000, New York, Random House, 1987.

Kissinger, Henry, Diplomacy, New York, Simon \& Schuster Paperbacks, 1994. Mearsheimer, John J., The tragedy of great power politics (Updated edition), New York, W. W. Norton \& Company, 2014.

Miall, Hugh; Ramsbotham, Oliver; Woodhouse, Tom, Contemporary conflict resolution, Cambridge, Polity Press, 2005. UNITED NATIONS. History of Peacekeeping in http://www.un.org/en/peacekeeping/operations/ history.shtml 
Obama, Barack, Haiti and America, "Newsweek", (25/01/2010), págs. 19-20.

OUR GLOBAL NEIGHBOURHOOD. Retport of the commission on global governance. Oxford: Oxford University Press, 1995.

Pereira, Chyara Sales; Souza, Leonardo César; Santos Filho, Onofre dos, Governança global, governamentalidade e uma nova visão acerca da relação entre explicação e compreensão nas Relações Internacionais, $3^{\circ}$ Encontro Nacional Associação Brasileira de Relações Internacionais, 2011.

Remarks by the President to Parliament in London, United Kingdom, May 25, 2011.

Rosenau, James \& Czempiel, Ernst-Otto (Org), Governance without government: order and change in world politics, Cambridge, Cambridge University Press, 1992.

S/2004/300 - Report of the Secretary-General on Haiti. United Nations, 16 de abril de 2004.

S/2004/300 - Report of the Secretary-General on Haiti. United Nations, 16 de abril de 2004.

S/2004/300 - Report of the Secretary-General on Haiti. United Nations, 16 de abril de 2004. p. 1.

S/2004/300 - Report of the Secretary-General on Haiti. United Nations, 16 de abril de 2004. pp. 20-34.

S/2004/300 - Report of the Secretary-General on Haiti. United Nations, 16 de abril de 2004. pp. 12-13.

$\mathrm{S} / 2004 / 698$ - Interim report of the Secretary-General on the United Nations Stabilization Mission in Haiti. United Nations, 30 de agosto de 2004.

S/2004/698 - Interim Report of the Secretary-General on the United Nations

Stabilization Mission in Haiti. United Nations, 30 de agosto de 2004.

S/2004/908 - Report of the Secretary-General on the United Nations

Stabilizations Mission in Haiti. United Nations, 18 de novembro de 2004.

S/2005/124 - Report of the Secretary-General on the United Nations

Stabilizations Mission in Haiti. United Nations, 25 de fevereiro de 2005.

$\mathrm{S} / 2005 / 313$ - Report of the Secretary-General on the United Nations

Stabilizations Mission in Haiti. United Nations, 13 de maio de 2005.

$\mathrm{S} / 2005 / 631$ - Report of the Secretary-General on the United Nations

Stabilizations Mission in Haiti. United Nations, 23 de junho de 2005.

S/2006/1003 - Report of the Secretary-General on the United Nations

Stabilizations Mission in Haiti. United Nations, 19 de dezembro de 2006.

$\mathrm{S} / 2006 / 592$ - Report of the Secretary-General on the United Nations

Stabilizations Mission in Haiti. United Nations, 28 de julho de 2006.

$\mathrm{S} / 2006 / 60$ - Report of the Secretary-General on the United Nations

Stabilizations Mission in Haiti. United Nations, 2 de fevereiro de 2006. 
S/2007/503 - Report of the Secretary-General on the United Nations

Stabilizations Mission in Haiti. United Nations, 22 de agosto de 2007.

S/2008/202 - Report of the Secretary-General on the United Nations

Stabilizations Mission in Haiti. United Nations, 26 de março de 2008.

S/2008/586 - Report of the Secretary-General on the United Nations

Stabilizations Mission in Haiti. United Nations, 27 de agosto de 2008.

S/2009/129 - Report of the Secretary-General on the United Nations

Stabilizations Mission in Haiti. United Nations, 6 de março de 2009.

S/2009/439 - Report of the Secretary-General on the United Nations

Stabilizations Mission in Haiti. United Nations, $1^{\circ}$ de setembro de 2009.

S/2010/200 - Report of the Secretary-General on the United Nations

Stabilizations Mission in Haiti. United Nations, 22 de fevereiro de 2010.

S/2010/200 - Report of the Secretary-General on the United Nations Stabilization

Mission in Haiti. United Nations, 22 de fevereiro de 2010. pp. 1-3.

S/2010/200 - Report of the Secretary-General on the United Nations Stabilization

Mission in Haiti. United Nations, 22 de fevereiro de 2010. p. 2.

S/2010/446 - Report of the Secretary-General on the United Nations

Stabilizations Mission in Haiti. United Nations, $1^{\circ}$ de setembro de 2010.

S/2011/540 - Report of the Secretary-General on the United Nations

Stabilizations Mission in Haiti. United Nations, 24 de março de 2011.

S/2012/678 - Report of the Secretary-General on the United Nations

Stabilizations Mission in Haiti. United Nations, 29 de fevereiro de 2012.

S/2013/139 - Report of the Secretary-General on the United Nations

Stabilizations Mission in Haiti. United Nations, 8 de março de 2013.

S/2013/493 - Report of the Secretary-General on the United Nations

Stabilizations Mission in Haiti. United Nations, 31 de agosto de 2012.

S/2013/493 - Report of the Secretary-General on the United Nations

Stabilizations Mission in Haiti. United Nations, 19 de agosto de 2013.

S/2014/162 - Report of the Secretary-General on the United Nations

Stabilizations Mission in Haiti. United Nations, 7 de março de 2014.

UNITED NATIONS. Peace and Security. In: http://www.un.org/en/ peacekeeping/operations/peace.shtml

UNITED NATIONS. United Nations Peacekeeping Operations: Principles and Guidelines.2008 in http://pbpu.unlb.org/pbps/Library/Capstone_ Doctrine_ENG.pdf

Zaum, Dominik, The Security Council, the general assembly, and war: the uniting for peace resolution in Vaughan Lowe; Adam Roberts; Jennifer Welsh; Dominik Zaum, The United Nations Security Council and War: the evolution of thought and practice since 1945, New York, Oxford University Press, 2008, págs 155-174. 